\title{
Model of the Correlation between Lidar Systems and Wind Turbines for Lidar Assisted Control
}

Schlipf, D.; Mann, Jakob; Rettenmeier, A.; Cheng, P.W.

Published in:

Extended Abstracts of Presentations from the 16th International Symposium for the Advancement of BoundaryLayer Remote Sensing

Publication date:

2012

Document Version

Publisher's PDF, also known as Version of record

Link back to DTU Orbit

Citation (APA):

Schlipf, D., Mann, J., Rettenmeier, A., \& Cheng, P. W. (2012). Model of the Correlation between Lidar Systems and Wind Turbines for Lidar Assisted Control. In Extended Abstracts of Presentations from the 16th International Symposium for the Advancement of Boundary-Layer Remote Sensing (pp. 210-213). Steering Committee of the 16th International Symposium for the Advancement of Boundary-Layer Remote Sensing.

\section{General rights}

Copyright and moral rights for the publications made accessible in the public portal are retained by the authors and/or other copyright owners and it is a condition of accessing publications that users recognise and abide by the legal requirements associated with these rights.

- Users may download and print one copy of any publication from the public portal for the purpose of private study or research.

- You may not further distribute the material or use it for any profit-making activity or commercial gain

- You may freely distribute the URL identifying the publication in the public portal 


\title{
Model of the Correlation between Lidar Systems and Wind Turbines for Lidar Assisted Control
}

\author{
D. Schlipf ${ }^{1}$, J. Mann ${ }^{2}$, A. Rettenmeier ${ }^{1}$, P. W. Cheng ${ }^{1}$ \\ 1 Stuttgart Wind Energy (SWE), University of Stuttgart, Germany \\ ${ }^{2}$ DTU Wind Energy, Risø Campus, Denmark
}

\begin{abstract}
Investigations for lidar assisted control to optimize the energy yield and to reduce loads of wind turbines increased significantly in recent years. For this kind of control it is crucial to know the correlation between the rotor effective wind speed and the wind preview provided by a nacelle or spinner based lidar system. If on the one side the assumed correlation is overestimated, the uncorrelated frequencies of the preview will cause unnecessary control action, inducing undesired loads. On the other side the benefits of the lidar assisted controller will not be fully exhausted, if correlated frequencies are filtered out.

To avoid these uncertainties, this work presents a method to model the correlation between lidar systems and wind turbines using Kaimal wind spectra. The derived model accounts for different measurement configurations and for different turbine sizes. The method is evaluated in two steps: At first the model is compared to the results from a lidar simulator to prove that the model is able to reproduce the effect of volume measurement, limited measurement points and scanning time. In a second step the model is augmented by a model for the decay due to wind evolution and compared to real measurement data with promising results. In addition an example is given, how this model can be used to design an optimal controller for a lidar system with fixed parameters and a given turbine and how the pattern of a scanning lidar system is optimized for a given turbine to improve the correlation.
\end{abstract}

\section{REQUIREMENTS FOR LIDAR ASSISTED CONTROL}

Reducing fatigue and extreme loads of the structure is an important design goal for large wind turbines control. Transient events such as gusts represent an unknown disturbance to the control system. Conventional feedback controllers can

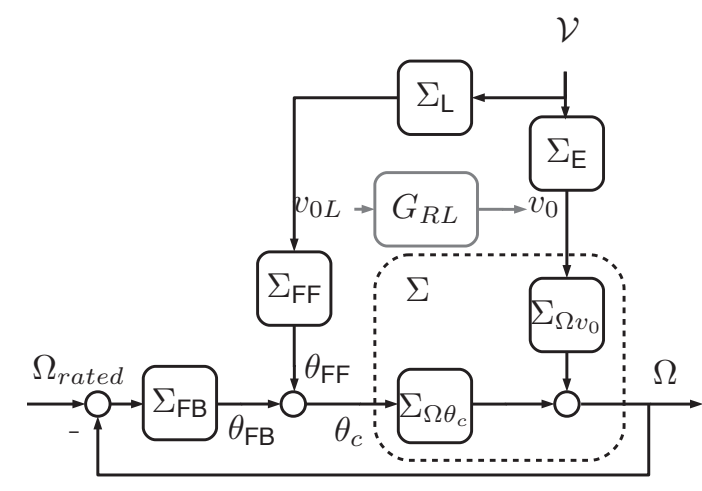

Figure 1: Feedforward control: The feedforward controller $\Sigma_{\mathrm{FF}}$ tries to compensate the effects of the wind field $\mathcal{V}$ to the rotor speed $\Omega$.

only provide delayed compensation for such excitations, since the disturbance effects must propagate through the entire wind turbine before showing its effects in the measured outputs. This usually results in additional loads for the wind turbine and requires high actuator rates for the disturbance compensation. Those effects can be avoided, if the wind ahead of the wind turbine is measured by remote sensing techniques such as lidar and the information is fed to the turbine controller.

The magnitude of load reduction depends on the quality of the wind preview expressed by the correlation of the Lidar measurements and the turbine reaction. In this section the requirements of the correlation for lidar assisted control are derived by a description of a feedforward collective pitch controller.

The feedforward controller (see Figure 1) is based on the work in [1] and combines the baseline feedback controller with a feedforward update. The main control goal of the collective pitch feedback controller $\Sigma_{\mathrm{FB}}$ is to maintain the rated rotor speed $\Omega_{\text {rated }}$. The system $\Sigma$ is disturbed by a wind field $\mathcal{V}$, which can be measured by a lidar system $\Sigma_{\mathrm{L}}$ in front of the turbine before reaching the rotor. If the wind would not change on its way $\left(\Sigma_{\mathrm{E}}=1\right)$ 
and in the case of perfect measurement the measured wind speed $v_{0 L}$ and the rotor effective wind speed $v_{0}$ are equal. The disturbance could be perfectly compensated by a feedforward controller $\Sigma_{\mathrm{FF}}=-\Sigma_{\Omega \theta_{c}}^{-1} \Sigma_{\Omega v_{0}}$, if the influence on the generator speed of the wind $\Sigma_{\Omega v_{0}}$ and the pitch angle $\Sigma_{\Omega \theta_{c}}$ is known and $\Sigma_{\Omega \theta_{c}}$ is invertible.

In reality $v_{0}$ cannot be measured perfectly due to the limitation of the lidar system $\Sigma_{L}$ and the wind evolution $\Sigma_{E}$. Therefore the needed feedforward controller is:

$$
\Sigma_{\mathrm{FF}}=-\Sigma_{\Omega \theta_{c}}^{-1} \Sigma_{\Omega v_{0}} \Sigma_{L}^{-1} \Sigma_{E} .
$$

Due to the interaction with the turbine and missing technology, modeling and verifying the wind evolution $\Sigma_{E}$ is very complicated. Also not all information of the wind field $\mathcal{V}$ can be reconstructed by the inverse of a real limited lidar system $\Sigma_{L}$. However, if the transfer function $G_{R L}$ from the measured wind speed to the rotor effective wind speed can be used to exploit all information captured by the lidar system:

$$
\Sigma_{\mathrm{FF}}=-\Sigma_{\Omega \theta_{c}}^{-1} \Sigma_{\Omega v_{0}} G_{R L} .
$$

For real time applications the transfer function $G_{R L}$ can be obtained from measurements and approximated by a standard low pass filter. Therefore the cut-off frequency $(-3 d B)$ of the corresponding filter can be considered as a quality criterion for the correlation. In the following sections a analytic way is presented to estimate the both the transfer function $G_{R L}$

$$
G_{R L}=\frac{S_{R L}}{S_{L L}},
$$

and the coherence $\gamma_{R L}$

$$
\gamma_{R L}=\frac{\left|S_{R L}\right|^{2}}{S_{R R} S_{L L}} .
$$

\section{ROTOR AVERAGED SPECTRUM}

The model of the rotor averaded spectrum $S_{v_{0} v_{0}}$ is derived from Kaimal wind specra. The hub height power spectral density of the longitudinal velocity component is given in [2] by the equation:

$$
\frac{f S_{H H, 1}(f)}{\sigma_{H H, 1}^{2}}=\frac{4 f L_{1} / v_{H H}}{\left(1+6 f L_{1} / v_{H H}\right)^{(5 / 3)}},
$$

where and $v_{H H}$ and $\sigma_{H H}$ are the mean and standard deviation of the longitudinal velocity component on hub height. The spatial correlation of a longitudinal velocity component with distance $r_{i j}$ of two points with coordinates $\left(y_{i}, z_{i}\right)$ and $\left(y_{j}, z_{j}\right)$ is defined as

$$
\gamma_{i j}(f)=\exp (-\underbrace{12 \sqrt{\left(f / v_{H H}\right)^{2}+\left(0.12 / L_{1}\right)^{2}}}_{\kappa} r_{i j})
$$

The rotor averaged spectrum can be derived by an average of the cross and auto spectrum densities of all points and combinations in the rotor plane $D$ with rotor radius $R$ :

$$
S_{R R}(f)=\frac{1}{\left(\pi R^{2}\right)^{2}} \int_{D_{j}} \int_{D_{i}} S_{i j}\left(r_{i j}, f\right) d y_{i} d z_{i} d y_{j} d z_{j}
$$

An explicit solution of (7) can be found showing the complexity by

$$
\begin{gathered}
S_{R R}(f)=2\left(R \kappa \left(-9 \pi_{0} \tilde{F}_{1}\left(; 2 ;(R \kappa)^{2}\right)-\right.\right. \\
\left.6 \pi \boldsymbol{L}_{2}(2 R \kappa)-8 R \kappa+6 \pi I_{0}(2 R \kappa)+3 \pi\right)+ \\
\left.3 \pi \boldsymbol{L}_{1}(2 R \kappa)\right) /\left(3 \pi(R \kappa)^{3}\right) S_{H H, 1}(f)
\end{gathered}
$$

where ${ }_{0} \tilde{F}_{1}$ is the regularized confluent hypergeometric function, $\boldsymbol{L}_{n}$ is the modified Struve function, and $I_{0}$ is the modified Bessel function of the first kind.

The discrete form of the averaged rotor spectrum can be calculated by with $n$ points inside the rotor area

$$
S_{R R}(f)=\frac{S_{H H, 1}(f)}{n^{2}} \sum_{i=1}^{n} \sum_{j=1}^{n} \gamma_{i j}(f) .
$$

\section{CORRELATION OF A PERFECT STARRING LIDAR}

Ignoring the filtering effect of a lidar, assuming Taylor's Hypothesis to be fully valid (no wind evolution), the spectrum of a starring lidar can be modeled by

$$
S_{L L}(f)=S_{H H, 1}(f) .
$$

Then the cross spectrum between the starring lidar and the rotor is

$$
S_{R L}(f)=\frac{1}{\pi R^{2}} \int_{D} S_{H j}\left(r_{H j}, f\right) d y_{j} d z_{j},
$$

where $S_{H j}$ means the cross spectrum of the hub and the point $j$ with distance $r_{H j}$.

This can be solved with

$$
\begin{aligned}
S_{R L}(f) & =\frac{S_{H H, 1}(f)}{\pi R^{2}} \int_{0}^{2 \pi} \int_{0}^{R} r \gamma_{H j}(f) d r d \phi \\
& =\frac{2 S_{H H, 1}(f)}{R^{2} \kappa^{2}}\left(1-\frac{R \kappa+1}{\exp (R \kappa)}\right) .
\end{aligned}
$$

With (3), (4) and

$$
k=\frac{2 \pi f}{\bar{u}}
$$

the transfer function $G_{R L}$ and the coherence $\gamma_{R L}$ can be calculated over the wavenumber $k$, independent of the mean wind speed $\bar{u}$. 


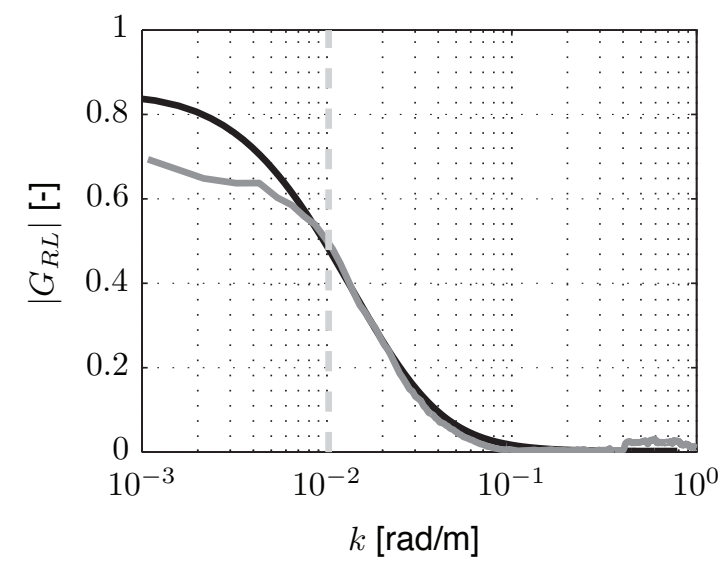

Figure 2: Transfer function for a nacelle anemometer and a turbine with $D=116 \mathrm{~m}$ : analytic (black) and measured (gray). Maximum wavenumber $\hat{k}=0.01 \mathrm{rad} / \mathrm{m}$ (dashed).

This model cannot be evaluated with a real lidar system, because wind evolution and volume measurement cannot be neglected. Therefore an anemometer on a $5 \mathrm{MW}$ turbine is compared to a nacelle anemometer. Figure 2 shows that the modeled transfer function fits to the data.

\section{CORRELATION OF A REAL SCANNING LIDAR}

For real scanning lidar system the model has to be extended by a model for the volume measurement and wind evolution. Here the model [3] is used.

To model the volume measurement, a Gaussian shape weighting function $f_{L}(a)$ depending on the distance $a$ to the focus point with full width at half maximum (FWHM) of $W=30 \mathrm{~m}$ is used, following the considerations of [4], [5] and [6]:

$f_{L}(a)=\frac{e^{-4 \ln 2(a / W)^{2}}}{\int_{-\infty}^{\infty} e^{-4 \ln 2(a / W)^{2}} d a}=\frac{2 \ln 2 e^{-4 \ln 2(a / W)^{2}}}{W \sqrt{\ln 2 \pi}}$.

With the weighting function it is possible to calculate the line-of-sight wind speed of each focus point with $f_{L}(a)$ by

$$
v_{\text {los }}=\int_{-\infty}^{\infty}\left(l_{x} u(a)+l_{y} v(a)+l_{z} w(a)\right) f_{L}(a) d a
$$

where $\left[\begin{array}{lll}l_{x} & l_{y} & l_{z}\end{array}\right]^{T}$ is the normalized laser beam vector and $\left[\begin{array}{lll}u(a) & v(a) & w(a)\end{array}\right]^{T}$ the wind vector at the distance $a$ to the focus point.

As LIDAR systems measure only the wind speed in line-of-sight direction, the three dimensional wind vector is reconstructed using the assumption

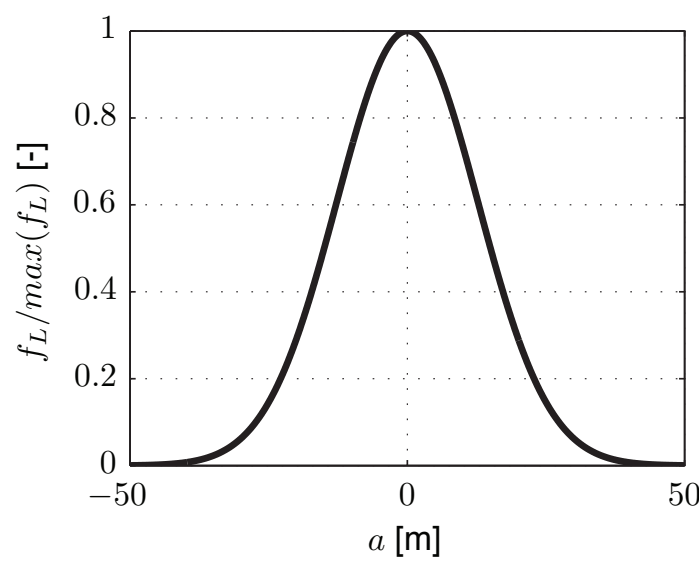

Figure 3: Normalized range weighing function $f_{L}(a)$ for the considered LIDAR system.

of perfect yaw alignment with the wind direction. If the turbine is perfectly aligned with the wind, the estimated lateral and vertical wind components are assumed to be zero and the longitudinal component $\hat{u}_{i_{f p}}$ for each focus point can be calculated as

$$
\hat{u}_{i_{f p}}=v_{l o s} / l_{x} .
$$

For each distance $i_{f d}$ the longitudinal wind component $\hat{u}_{i_{f p}}$ is then averaged over the $n_{f p}$ focus points of the last trajectory by

$$
v_{i_{f d}}=\sum_{i_{f p}}^{n_{f p}} \hat{u}_{i_{f p}} / n_{f p}
$$

for a rotor effective value and the obtained time series of the measurements $v_{i_{f d}}$ is time-shifted according to Taylor's frozen turbulence hypothesis and combined to the rotor effective wind speed:

$$
v_{0 L}=\sum_{i_{f d}}^{n_{f d}} v_{i_{f d}} / n_{f d}
$$

The spectrum of a scannig lidar can then be found by a Fourier transform of (18), including (17), (16) and (15) using a weighting function such as (14) and a wind evolution model such as [3].

\section{LIDAR SYSTEM OPTIMIZATION}

The proposed model can be used to optimize a lidar system. To determine the optimum correlation of a lidar system with three independent beams on a turbine with $D=40 \mathrm{~m}$, the measurement distance $x$ and the scan radius $r$ is varied, see Figure 5 . The value of maximum wavenumber $\hat{k}$ at $-3 d B$ below the steady value of the transfer 


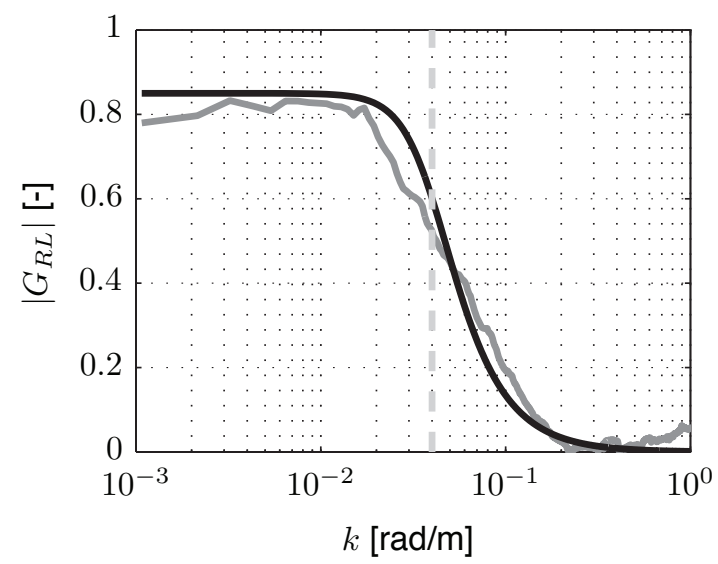

Figure 4: Transfer function for a scanning lidar and a turbine with $D=116 \mathrm{~m}$ : analytic (black) and measured (gray). Maximum wavenumber $\hat{k}=$ $0.04 \mathrm{rad} / \mathrm{m}$ (dashed).

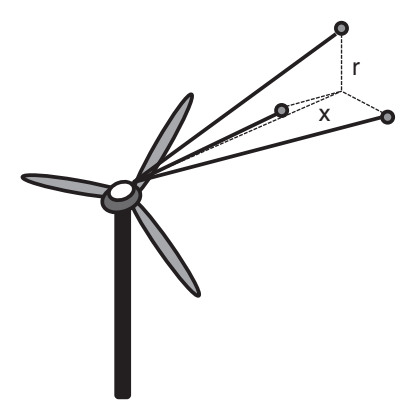

Figure 5: Scope of lidar with three beams.

function is shown in Figure 6. A optimum value $(\hat{k}=0.03 \mathrm{rad} / \mathrm{m})$ can be found at $x=40 \mathrm{~m}$ and $r=12 m$.

\section{CONCLUSION AND OUTLOOK}

This work presents a model to estimate the correlation between a lidar and a turbine, considering volume measurements, different scanning patterns and wind evolution. A criterion for lidar assisted control is derived: for a collective pitch feedforward controller the transfer function from the measured wind speed to the rotor effective wind speed obtained from turbine data has to be used to filter the data. For real application this transfer function has to be fitted to a standard filter and therefore the $-3 d B$ of considered as a useful criterion for the the correlation between a lidar and a turbine.

The model can be used to optimize the configuration of a lidar system such as probe volume and scan pattern.

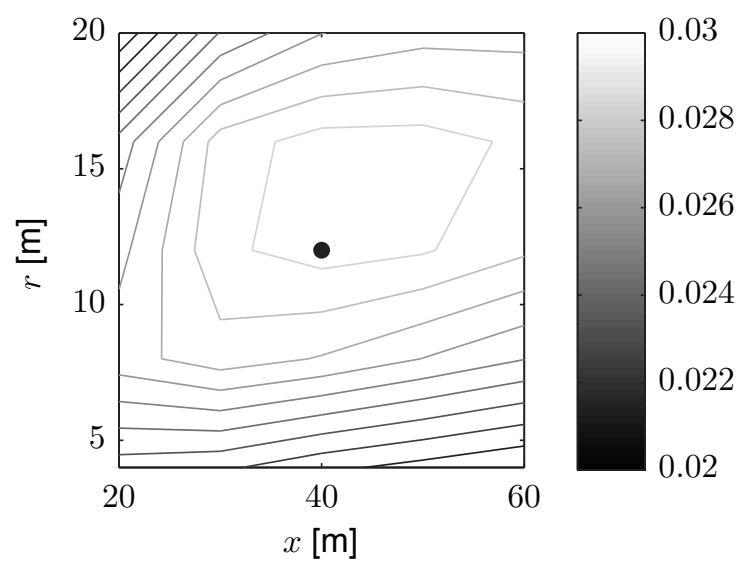

Figure 6: Maximum wavenumber of lidar with three beams on a turbine with $D=40 \mathrm{~m}$ (dashed) and different distances and radii.

\section{ACKNOLEGEMENT}

Part of this research is funded by the German Federal Ministry for the Environment, Nature Conservation and Nuclear Safety (BMU) in the framework of the German joint research project "LIDAR II - Development of nacelle-based lidar technology for performance measurement and control of wind turbines" (FKZ 0325216B).

\section{References}

[1] D. Schlipf, T. Fischer, C. E. Carcangiu, M. Rossetti, and E. Bossanyi, "Load analysis of lookahead collective pitch control using LiDAR," in Proc. DEWEK, 2010.

[2] IEC 61400-1 third edition 2005-08 Wind turbines - Part 1: Design requirements, IEC Std., Rev. third edition, 2005.

[3] L. Kristensen, "On longitudinal spectral coherence," Boundary-Layer Meteorology, vol. 16, pp. 145-153, 1979.

[4] J. P. Cariou, "Pulsed lidars," in Remote Sensing for Wind Energy. Risøreport Risø-I3184(EN). A. Peña and C. B. Hasager, June 2011, pp. 65-81, risø-DTU.

[5] P. Lindelöw, "Fiber based coherent lidars for remote wind sensing," Ph.D. dissertation, Technical University of Denmark, 2008.

[6] V. A. Banakh and I. N. Smalikho, "Estimation of the turbulence energy dissipation rate from the pulsed doppler lidar data," Atmospheric and Oceanic Optics, vol. 10, pp. 957-965, 1997. 